\title{
INTRAVENOUS IMMUNOGLOBULIN FOR PYODERMA GANGRENOSUM ASSOCIATED WITH CUTANEOUS MYCOBACTERIAL INFECTION
}

Lívia Airoldi Rodrigues ${ }^{1, *}$, Carolina Carotenuto Ramos ${ }^{1}$, Giovanna Paliares Monteiro ${ }^{1}$, Aldrei Costa Araújo1, Jean Marcos de Souza ${ }^{1,2}$

1.Universidade Cidade de São Paulo, São Paulo (SP), Brazil. 2.Universidade de São Paulo, São Paulo (SP), Brazil.

*Corresponding author: airoldilivia@gmail.com

\section{BACKGROUND}

Pyoderma gangrenosum (PG) is a rare idiopathic neutrophilic dermatosis. It is associated with an abnormal immune response that causes chronic skin ulcers, usually accompanied by other inflammatory comorbidities (e.g., inflammatory bowel disease, arthritis and malignancies). It is characterized by painful and purulent ulcerated lesions of rapid evolution; lower limbs are more affected. With no pathognomonic exams available, clinical history, complete physical examination of ulcers and skin biopsy are important for diagnosis. Treatment is done with immunosuppressants, but concomitant infection might preclude them. We report the rare association of PG concomitant with mycobacterium infection and the utility of intravenous immunoglobulin as an immunomodulatory agent.

\section{CASE REPORT}

A61-year-old woman with longstanding seropositive rheumatoid arthritis (RA), diabetes and hypertension was admitted with a history of painful purulent ulcerative lesion on right supramalleolar lower limb for three months (Fig. 1A). She was treating RA with leflunomide, abatacept and prednisone ( $20 \mathrm{mg} /$ day). Multiple antibiotics had been unsuccessful so far. The ulcer internal border was biopsied, presenting lobular panniculitis with neutrophil infiltration, necrosis and no granuloma formation. Both clinical presentation and biopsy results were PG compatible. A magnetic resonance imaging (MRI) was performed and depicted a profound abscess (Fig. 2) that was further aspirated and Acid-Fast Bacilli was detected through microscopic examination. Specific bacteria could not be isolated, probably because the patient was already in use of antibiotics (i.e., quinolone). The diagnostic hypothesis was PG decompensated by rapidly growing mycobacteria. All immunosuppressants were, thus, suspended. She was treated for mycobacterium with RIPE therapy, levofloxacin and azithromycin for 2 months. The patientalso had 8 monthly applications of intravenous immunoglobulin ( $2 \mathrm{~g} / \mathrm{kg}$ per month) for PG treatment, associated with prednisone $20 \mathrm{mg} /$ day that was slowly tapered. An important clinical improvement was shown, with resolution of the ulcer and the abscesses. As the ulcer is currently healed (Fig. 1B), medical follow-up concentrates on controlling rheumatoid arthritis with anti-TNF medication (etanercept), free from corticosteroids.

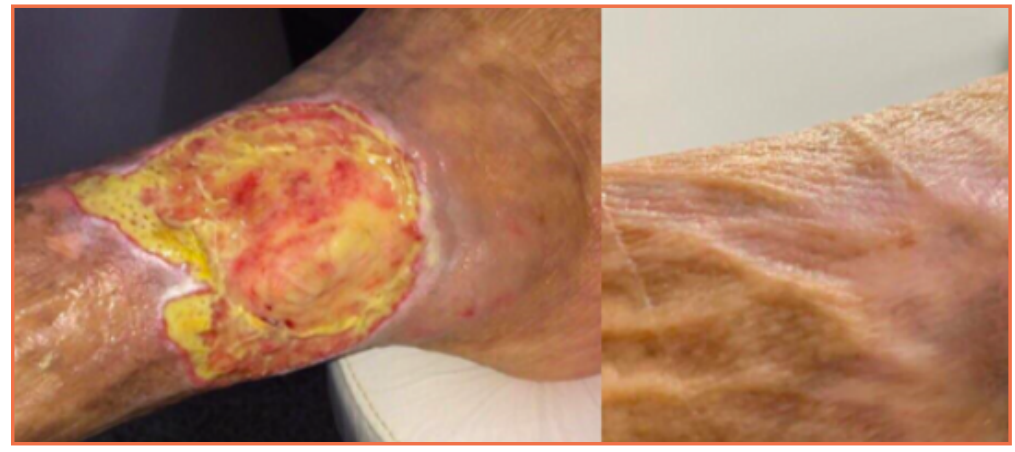

Figure 1. (A) Purulent ulcerative lesion on right limb. (B) Ulcer scar after treatment.

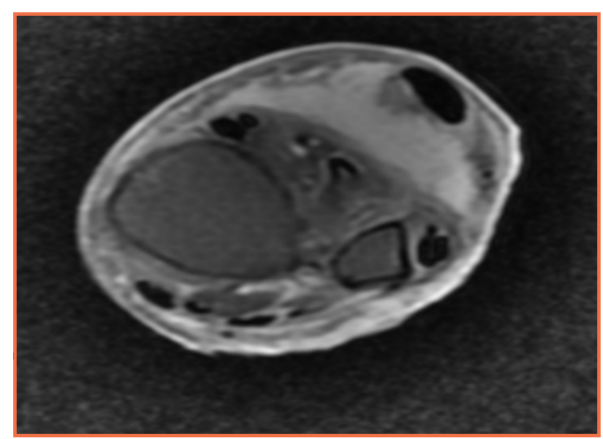

Figure 2. T2-weighted MRI showing the leg abscess, anterior to the Achilles tendon.

\section{CONCLUSION}

This case-report shows a correlation between PG and mycobacterium and presents an alternative, effective treatment with immunoglobulin.

\section{ACKNOWLEDEGMENTS}

The patient provided written informed consent for all the data utilized. 